\title{
Modelamiento de la Viscosidad con Base en una Ecuación Cúbica $\mu$ TP del Tipo Peng-Robinson
}

\author{
Luis F. Cardona, Luis A. Forero y Jorge A. Velásquez* \\ Facultad de Ingeniería Química, Departamento de Ingeniería, Grupo de Investigación en Pulpa y Papel, Universidad \\ Pontificia Bolivariana, Circular 1ª \# 70-01, Medellín-Colombia, (e-mail: jorge.velasquezj@upb.edu.co). \\ * autor a quien debe ser dirigida la correspondencia
}

Recibido Nov. 19, 2018; Aceptado Ene. 28, 2019; Versión final Feb. 22, 2019, Publicado Ago. 2019

\begin{abstract}
Resumen
Se aplica un modelo para el cálculo de la viscosidad del tipo ecuación cúbica de estado de Peng-Robinson. El modelo ha sido empleado para correlacionar y predecir viscosidades de sustancias puras y mezclas en especial en la región de saturación. Los parámetros del modelo han sido generalizados en términos de la masa molar. Las expresiones generalizadas son estimadas mediante el ajuste de datos experimentales de alcanos y alcoholes. Las desviaciones absolutas promedio para la viscosidad, entre los valores correlacionados y los experimentales, son $6.26 \%$ y $7.21 \%$ para alcanos y alcoholes, respectivamente. Se realizan cálculos predictivos con desviaciones absolutas promedio de $3.99 \%$ para alcanos y $13.76 \%$ para alcoholes. Para viscosidades de mezclas se utilizaron tres enfoques de reglas de mezclado. Las desviaciones promedio calculadas son $18.66 \%, 10.31 \%$ y $3.85 \%$ para las reglas simples, de uno y dos parámetros respectivamente. Los resultados indican que el modelo propuesto proporciona resultados adecuados y consistentes, teniendo en cuenta la simplicidad de las expresiones generalizadas desarrolladas.
\end{abstract}

\section{Modeling the Viscosity Based on Peng-Robinson Type Cubic $\mu$ TP Equation}

\begin{abstract}
A viscosity model based on the Peng-Robinson cubic equation of state is applied. The model has been used to correlate and predict viscosities of pure substances and mixtures especially in the saturation zone. The parameters of the model are generalized in terms of the molar mass. The generalized expressions are estimated by fitting the experimental data for a group of alkanes and alcohols. Average deviations between calculated values and experimental data for alkanes and alcohols are $6.26 \%$ and $7.21 \%$. Some predictive calculations are performed for alkanes and alcohols finding average absolute deviation of $3.99 \%$ and $13.76 \%$. To extend the model to mixtures, some binary mixtures calculations are performed using three mixing rule approaches. The results obtained with the simple, one and two interaction parameters mixing rules are $18.66 \%, 10.31 \%$ and $3.85 \%$. The results indicate that the new viscosity model provides results that can be considered adequate and consistent taking in account the simplicity of the generalized expressions.
\end{abstract}

Keywords: viscosity; cubic equation of state; pure substances; mixtures 


\section{INTRODUCCIÓN}

La viscosidad es una propiedad de transporte utilizada en aplicaciones de la industria química, como la agitación, el mezclado, la filtración, el intercambio de calor, la concentración y el dimensionamiento de las zonas de reacción y separación (Shukla et al., 2007; Ortega et al., 2016; Márquez-Baños et al.; 2016). Por esta razón su predicción tanto en sistemas líquidos como gaseosos en amplios rangos de temperatura y presión son de relevancia para el desarrollo y optimización de procesos industriales (Xuan et al., 2007; Qunfang y Yu-Chun, 1999; Fernández et al., 2004). Cuando se carece de datos experimentales, las propiedades de transporte pueden estimarse mediante diferentes metodologías entre las que se destacan los métodos empíricos, las correlaciones que utilizan el principio de los estados correspondientes (Poling et al., 2001), modelos análogos a la ecuación de Arrhenius como la teoría de Eyring (Shukla et al., 2007; García et al., 2007), modelos teóricos o semi-teóricos de viscosidad acoplados a una ecuación de estado como la teoría de fricción (Quiñones-Cisneros et al., 2003), la teoría de entropía residual (Novak, 2013), la teoría del volumen libre (Llovell et al., 2013) y otros modelos basados en la similitud geométrica entre los diagramas PV(volumen molar) $T$ y $P \mu$ (viscosidad)T. En el último tipo de modelos, se describe la viscosidad como una ecuación cúbica de estado mediante un solo modelo independiente, válido para amplios rangos de temperatura y presión en sistemas líquidos y gaseosos (Guo et al., 1997; Fan y Wang, 2006).

Little y Kennedy (1968) desarrollaron el primer modelo de viscosidad mediante analogía con la ecuación de estado (EoS) de van der Waals. Los autores estimaron las constantes de la EoS por medio del ajuste de 1006 datos experimentales de hidrocarburos, reportando desviaciones del 1.9\%. Posteriormente, Lawal (1986) propuso un modelo de ecuación de estado para la viscosidad con cuatro parámetros, aplicable a hidrocarburos puros y mezclas en un rango de temperatura de $-183^{\circ} \mathrm{F}$ a $482{ }^{\circ} \mathrm{F}$ y una presión superior a 20000 psi con desviaciones del 5.9\%. Por otro lado, Guo et al. (1997) desarrollaron dos modelos de viscosidad basados en las EoSs de Peng-Robinson (PR) y Patel-Teja (PT) para 24 hidrocarburos y dióxido de carbono obteniendo desviaciones promedio para PR de $6.18 \%$ y PT de $15.07 \%$. Los autores reportaron desviaciones promedio de $7.2 \%$ para mezclas. Además, Guo et al. (2001) desarrollaron una EoS del tipo PR para 24 sustancias no polares, principalmente hidrocarburos con desviaciones promedio del 7.0\%. En el 2006, Fan y Wang desarrollaron un modelo basado en la EoS de PR para el cálculo de la viscosidad de 10 hidrocarburos y sus mezclas, con porcentajes de desviación promedio para sustancias puras del $12.01 \%$ y de $8.33 \%$ para mezclas (Fan y Wang, 2006). En el 2014, Wu et al., modificaron el modelo de EoS propuesto por Fan y Wang (2006), con porcentajes de desviación promedio del $4.78 \%$ cuando se aplica en 12 hidrocarburos, dióxido de carbono y nitrógeno. Bonyadi y Rostami (2017) usaron el modelo basado en la EoS de Soave-Redlich-Kwong para realizar estimaciones de la viscosidad de hidrocarburos, nitrógeno y dióxido de carbono con desviaciones promedio de $7.80 \%$. Recientemente, Khosharay et al. (2017) estima viscosidades de refrigerantes y sus mezclas usando la EoS de Patel-Teja con desviaciones promedio de $7.40 \%$ para sustancia pura y $3.50 \%$ para mezclas.

Debido a que los modelos de viscosidad tipo EoS disponibles en la literatura han sido poco aplicados a sustancias polares, en el presente trabajo se desarrolla un modelo para el cálculo de la viscosidad de sustancias polares y no polares con sus mezclas tanto en fase líquida como en vapor especialmente en el estado saturado. El modelo se desarrolla mediante similitud con la ecuación cúbica de estado de PengRobinson (PR). Los parámetros se determinan inicialmente mediante el ajuste de los datos experimentales de viscosidad en la región de equilibrio líquido-vapor. El desempeño del modelo es comparado con aquel que se obtiene con correlaciones disponibles en la literatura para fase líquida (Letsou y Stiel, 1973; Mehrotra, 1991; Poling et al., 2001; Gardas y Coutinho, 2008) o para fase vapor (Chung et al., 1988; Poling et al., 2001; Satyro y Yarranton, 2010; Poling et al., 2001). Además, para sistemas binarios se evalúan tres enfoques que incluyen la regla de mezcla simple (Fan y Wang, 2006) y reglas de mezcla con uno y dos parámetros de interacción (Khosharay et al., 2015; Teja y Rice, 1981).

\section{MODELO DE VISCOSIDAD BASADO EN LA ECUACIÓN DE ESTADO DE PENG-ROBINSON (PR $\mu 0)$}

A partir de la similitud entre el diagrama termodinámico $P V($ volumen molar $) T$ y $T \mu$ (viscosidad)P es posible desarrollar un modelo de viscosidad de la siguiente manera (Fan y Wang, 2006; Guo et al., 1997): 1) Las posiciones de $T$ y $P$ de la EoS son intercambiadas; 2) $V$ es reemplazada por $\mu$; 3) la constante de los gases $R$ se reemplaza por una equivalente $R^{\prime}$. A partir de la EoS de Peng-Robinson (PR) (Peng y Robinson, 1976) se tiene que:

$$
T^{\prime}=\frac{R^{\prime} P}{\mu-b}-\frac{a \beta}{\mu^{2}+2 \mu b-b^{2}}
$$

Donde el término $T^{\prime}$ 'se define como: $T^{\prime}=\left|T-T_{d}\right|$ y $T_{d}=0.45 \cdot T_{c}$ (Fan y Wang, 2006). En la ecuación $1, P$ denota la presión en bar; $T$ la temperatura en kelvin; $\mu$ la viscosidad en $\mu \mathrm{P}$ (micropoise) y los subíndices $c$ denotan el 
estado crítico. El coeficiente 0.45 corresponde a un valor recomendado en la literatura (Fan y Wang, 2006). Finalmente, los parámetros se definen como:

$$
\begin{aligned}
& R^{\prime}=\alpha(P) \cdot r_{c} \\
& a_{c}=0.45724 \frac{r_{c}^{2} P_{c}^{2}}{T_{c}^{\prime}} \\
& b_{c}=0.077796 \frac{r_{c} P_{c}}{T_{c}^{\prime}} \\
& r_{c}=\frac{T_{c}^{\prime} \mu_{c}}{Z_{c} P_{c}}
\end{aligned}
$$

De forma similar a la función $\alpha(T)$ de las ecuaciones $P V T$, la función $\alpha(P)$ debe tener un valor unitario a la presión crítica, es decir: $\alpha\left(P_{r}=1\right)=1$, donde $P_{r}$ es la presión reducida. En el presente trabajo se utiliza la siguiente expresión para el cálculo de $\alpha(P)$ :

$$
\alpha=P_{r}^{-1}+e_{1} \cdot P_{r}^{-1} \cdot\left[\left(P_{r}+0.25\right)^{-1}-0.8\right]+e_{2} \cdot\left(1-P_{r}^{-1}\right)
$$

La ecuación 6 es la función propuesta por Fan y Wang (2006). Los valores de $e_{1}$ y $e_{2}$, son parámetros que se deben determinar para cada sustancia. En la ecuación $5, \mu_{c}$ es la viscosidad crítica y es calculada mediante la correlación de Uyehara y Watson (Guo et al., 1997; Fan y Wang, 2006) y $Z_{c}$ es el factor de compresibilidad crítico. Por otro lado, la función $\beta$ (ver ecuación 1) es una expresión que se utiliza con el propósito de mejorar el desempeño del modelo en sustancias polares. Esta función debe tener un valor unitario a la temperatura y presión crítica. La ecuación para el cálculo de $\beta$ es:

$$
\beta=\exp \left[\beta_{1}\left(\sqrt{T_{r}}-1\right)\right]+\beta_{2}\left(\sqrt{P_{r}}-1\right)
$$

La ecuación 7 es la función propuesta por Guo et al. (2001). Los valores $\beta_{1}$ y $\beta_{2}$ son parámetros que se deben determinar para cada sustancia. En la ecuación $7, P_{r}$ es la presión reducida y $T_{r}$ es la temperatura reducida.

Selección de la raíz más apropiada: De acuerdo con las ecuaciones 1-7, para calcular la viscosidad con el modelo tipo EoS cúbica, se necesitan propiedades de las sustancias puras tales como la presión crítica, la temperatura crítica, el factor acéntrico, el factor de compresibilidad crítico. Los valores de las propiedades mencionadas se encuentran disponibles en la literatura (Daubert, 1998). Con las propiedades mencionadas anteriormente, se determina el estado de agregación. Posteriormente, se utiliza el método de Cardano (Jarrahian y Heidaryan, 2014) para calcular las raíces del modelo $P R \mu_{0}$. Las raíces son seleccionadas de acuerdo con los siguientes criterios: 1) en la región de vapor cuando la presión es menor o igual que la presión de saturación a la temperatura del sistema, se selecciona la raíz real menor positiva; 2) en la región de líquido cuando la presión es igual o mayor que la presión de saturación a la temperatura del sistema, se selecciona la raíz real mayor positiva; 3) en la región de saturación la raíz real mayor positiva corresponde al líquido saturado, mientras que la raíz real menor positiva es vapor saturado.

Corrección de la viscosidad en líquidos: Con el objetivo de mejorar el desempeño de los modelos en fase líquida es posible definir una corrección de viscosidad análoga al volumen trasladado que se usa en ecuaciones cúbicas PVT (Fan y Wang, 2006; Abudour et al., 2012; Nazarzadeh y Moshfeghian, 2013; Cardona-Palacio, 2016). La viscosidad corregida se define como:

$$
\mu=\mu^{M O D E L O}+c_{0}+c
$$

Donde $\mu^{\text {MODELO }}$ es la viscosidad obtenida luego de resolver la ecuación 1 . Esta viscosidad depende del estado de agregación en el que se encuentre la sustancia: líquido o vapor. En la ecuación $8, c_{0}$ es una función dependiente de la presión y se define así:

$$
c_{0}=a_{1} \cdot\left(P_{r}-1\right)+a_{2} \cdot\left[\left(P_{r}+1\right)^{-1}-0.5\right]
$$


Por otro lado, $c$ es un parámetro que depende de la viscosidad de la siguiente manera:

$$
c=v_{1} \cdot \ln \mu_{r}+v_{2} \cdot\left(\mu_{r}-1\right)+v_{3} \cdot\left[\left(\mu_{r}+1.25\right)^{-1}-0.4444\right]
$$

En la ecuación 10, $\mu_{r}$ es la viscosidad reducida, $\mu_{r}=\mu^{\text {MODELOO }} \mu_{c}$ y los valores de $a_{1}, a_{2}, v_{1}, v_{2}, v_{3}$ dependen de cada sustancia. Sus valores se determinan mediante el ajuste de datos experimentales en fase líquida. Las ecuaciones 9 y 10, son funciones matemáticas propuestas por Fan y Wang (2006).

Corrección de la viscosidad en gases: Para el caso de gases, la variable $c$ que aparece en la viscosidad corregida (ver ecuación 8) se define como:

$$
c=b_{1} \cdot\left(\mu_{r}-1\right)+b_{2} \cdot\left[\left(P_{r}+1\right)^{-1}-0.5\right]+\frac{b_{3} \cdot\left(\mu_{r}-1\right)}{\left(P_{r}+1\right)}
$$

Los valores de $b_{1}-b_{3}$ dependen de cada sustancia y son estimados mediante el ajuste de datos experimentales en fase vapor. La ecuación 11 es una modificación de la función propuesta por Fan y Wang (2006). El último término de la expresión se utiliza con el propósito de mejorar el desempeño del modelo en sustancias polares en fase gaseosa.

\section{DETERMINACIÓN DE PARÁMETROS}

La determinación de los parámetros del modelo requiere de datos experimentales para realizar el ajuste. Para alcanos se eligen los compuestos entre metano y decano y se utilizan los datos experimentales reportados y recomendados como datos de referencia por el webbook de la NIST (NIST, 2015; Linstrom et al., 2018). Para alcoholes se eligen compuestos entre metanol y 1-decanol. Adicionalmente, se incluyen otras sustancias nopolares y polares como benceno, penteno, dióxido de carbono, agua, acetona y ácido acético. Para todas las sustancias diferentes a los alcanos se emplean los datos reportados y recomendados como aceptados por la DIPPR (Daubert, 1998).

Todos los parámetros del modelo son estimados a partir de condiciones de saturación a temperaturas reducidas entre 0.5 y 0.8 . Para cada sustancia se toman 30 datos experimentales que incluyen 15 datos de líquido saturado y 15 datos de vapor saturado. En total 600 datos experimentales son utilizados en el proceso de ajuste. Inicialmente, con los datos experimentales de viscosidad en líquidos se determinan los valores de los parámetros: $e_{1}, e_{2}, \beta_{1}, \beta_{2}, a_{1}, a_{2}, v_{1}-v_{3}$. Además, con los datos experimentales de vapor se estiman los valores de $b_{1}-b_{3}$. El proceso de determinación de parámetros es realizado mediante regresión no lineal (Chapra y Canale, 2007; Cardona-Palacio, 2016) y el algoritmo de Lasdon et al. (1978) incorporado en Solver de MS Excel, con la siguiente función objetivo a minimizar:

$$
\text { F.O. }=|\% \Delta \mu|=\frac{100}{N} \cdot \sum_{i=1}^{N}\left|\frac{\mu_{\exp }-\mu_{c a l}}{\mu_{\exp }}\right|
$$

Donde $\mu_{\text {exp }}$ representa la viscosidad experimental, $\mu_{c a l}$ la viscosidad calculada por el modelo $P R \mu_{0}$ y $N$ es el número total de datos experimentales. En la Tabla 1, se reportan los parámetros estimados del modelo $P R \mu_{0}$. Es importante aclarar que $\beta_{1}$ no depende de la naturaleza de la sustancia. $\beta_{2}, a_{1}, a_{2}, e_{2}, v_{2}, v_{3}, b_{1}$ y $b_{3}$ tienen un valor definido si la sustancia es no polar o polar. Finalmente, $e_{1}, v_{1}$ y $b_{2}$ son específicos de cada sustancia. Sin embargo, para alcanos y alcoholes es posible correlacionar estos tres parámetros en términos del peso molecular, mediante la siguiente expresión:

$$
z_{i}=z_{1} M_{w}+z_{2}, z_{i}=e_{1}, v_{1}, b_{2}
$$

En la ecuación $13, z_{1}$ corresponde a la pendiente en $\mathrm{mol} / \mathrm{g}, z_{2}$ el intercepto y $M_{w}$ el peso molecular en $\mathrm{g} / \mathrm{mol}$. En la Tabla 2, se muestran los valores estimados de las constantes de la ecuación 13. Los coeficientes de determinación de los parámetros $e_{1}, v_{1}$ y $b_{2}$ son superiores a 0.8698 , para alcanos; mientras que para alcoholes son superiores a 0.7994 . 


\begin{tabular}{|c|c|c|c|c|c|c|c|c|c|c|c|c|c|c|c|c|c|c|c|c|c|c|c|c|c|c|}
\hline$\Omega$ & \multicolumn{13}{|c|}{ 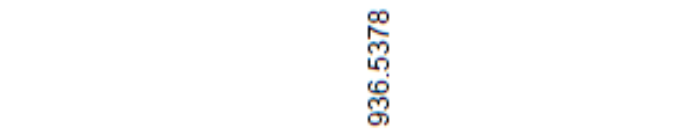 } & \multicolumn{13}{|c|}{ 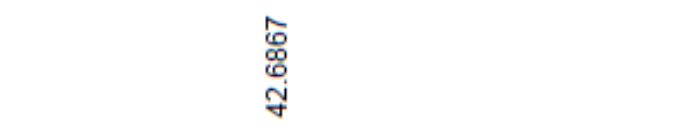 } \\
\hline$\approx$ & 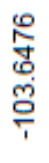 & 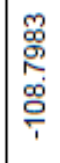 & 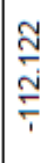 & 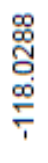 & 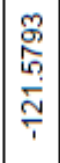 & $\begin{array}{l}\bar{\gamma} \\
\bar{\alpha} \\
\tilde{N} \\
\bar{N}\end{array}$ & 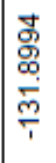 & $\begin{array}{l}\frac{F}{T} \\
\stackrel{M}{M} \\
\frac{m}{1}\end{array}$ & 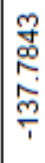 & ד্ণ & 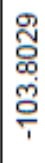 & 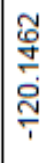 & 粱 & 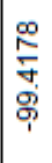 & 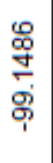 & 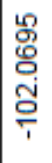 & 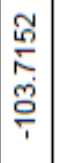 & 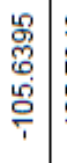 & 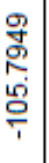 & $\begin{array}{l}\frac{m}{8} \\
8 \\
\infty \\
\frac{0}{1}\end{array}$ & 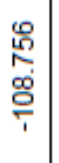 & 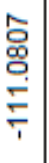 & 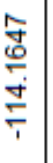 & $\begin{array}{l}\hat{D} \\
\stackrel{2}{0} \\
\stackrel{0}{0} \\
\stackrel{1}{1}\end{array}$ & 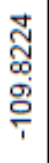 & 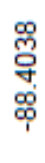 \\
\hline 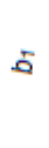 & \multicolumn{13}{|c|}{ 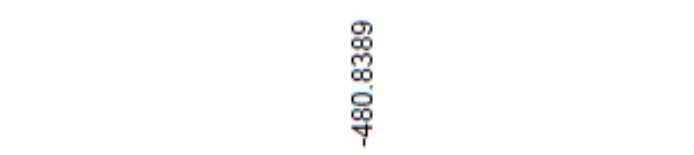 } & \multicolumn{13}{|c|}{$\begin{array}{l}\hat{\circ} \\
\frac{0}{0} \\
\text { pे }\end{array}$} \\
\hline$s$ & \multicolumn{13}{|c|}{ 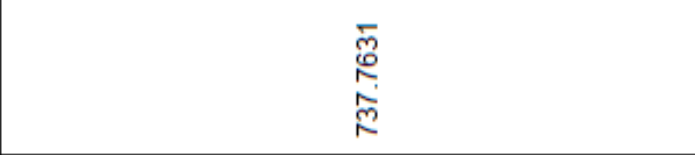 } & \multicolumn{13}{|c|}{ 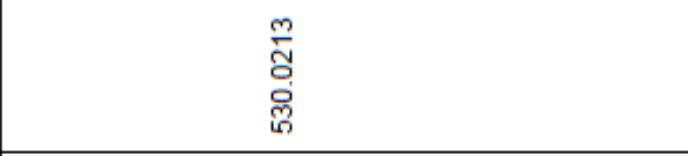 } \\
\hline$s^{s}$ & \multicolumn{13}{|c|}{$\begin{array}{l}\hat{o} \\
\text { ⿳亠口冋 } \\
\stackrel{+}{+}\end{array}$} & \multicolumn{13}{|c|}{ 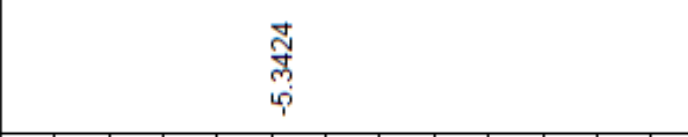 } \\
\hline 5 & 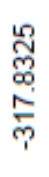 & $\begin{array}{l}\stackrel{2}{2} \\
\stackrel{S}{S} \\
\stackrel{\text { N }}{\text { ? }}\end{array}$ & 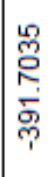 & 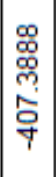 & 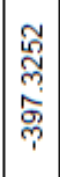 & 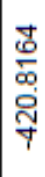 & 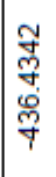 & 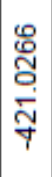 & $\begin{array}{l}\frac{\pi}{m} \\
\frac{m}{6} \\
\frac{0}{y}\end{array}$ & 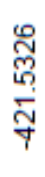 & 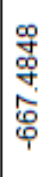 & 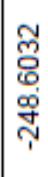 & 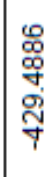 & 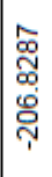 & 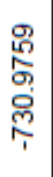 & 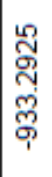 & 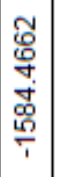 & $\begin{array}{l}0 \\
\stackrel{0}{0} \\
\stackrel{\tilde{ల}}{0} \\
\frac{0}{1}\end{array}$ & 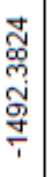 & 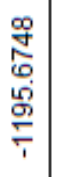 & 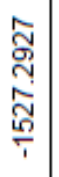 & 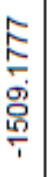 & 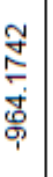 & 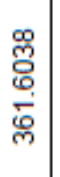 & 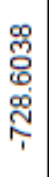 & 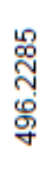 \\
\hline $\mathscr{\Phi}$ & \multicolumn{13}{|c|}{ 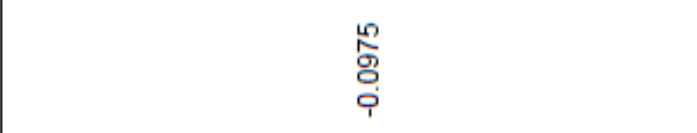 } & \multicolumn{13}{|c|}{ 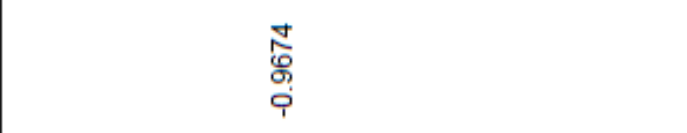 } \\
\hline$\Phi$ & 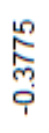 & $\begin{array}{l}\text { ợ } \\
\text { ơ } \\
\text { co }\end{array}$ & 究 & 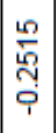 & 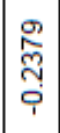 & $\begin{array}{l}\text { त्र̃ } \\
\text { ஸे }\end{array}$ & $\frac{\mathfrak{n}}{\stackrel{2}{2}}$ & 草 & $\frac{\infty}{c}$ & $\begin{array}{l}\frac{m}{\circ} \\
\text { ô } \\
\end{array}$ & $\frac{n}{\frac{m}{0}}$ & 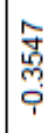 & 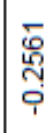 & 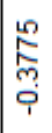 & $\begin{array}{l}\hat{\bar{o}} \\
\overline{0} \\
\bar{i}\end{array}$ & 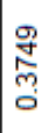 & 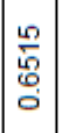 & $\begin{array}{l}\mathscr{0} \\
0 \\
0 \\
0\end{array}$ & مُ & $\begin{array}{l}\text { ôे } \\
\text { पे } \\
0\end{array}$ & 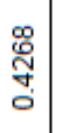 & 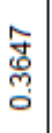 & 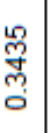 & $\begin{array}{l}0 \\
0 \\
0 \\
0 \\
0 \\
0 \\
0\end{array}$ & 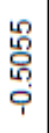 & 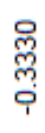 \\
\hline ฮี & \multicolumn{13}{|c|}{ 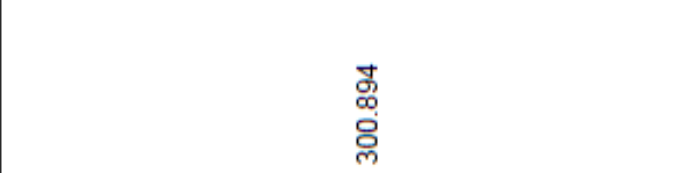 } & \multicolumn{13}{|c|}{ 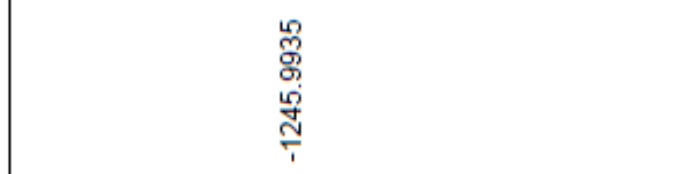 } \\
\hline ฮె & \multicolumn{13}{|c|}{ 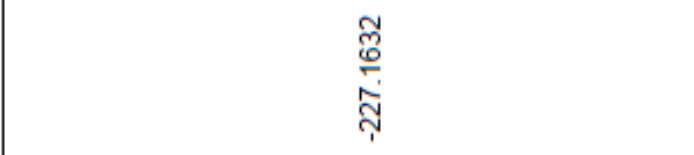 } & \multicolumn{13}{|c|}{ 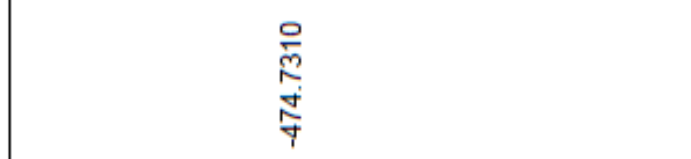 } \\
\hline \& & \multicolumn{13}{|c|}{ 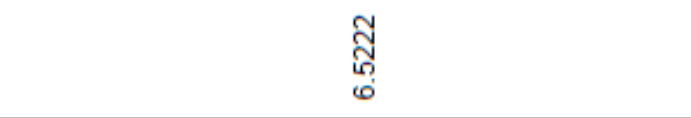 } & \multicolumn{13}{|c|}{ 命 } \\
\hline 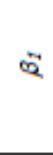 & \multicolumn{26}{|c|}{ 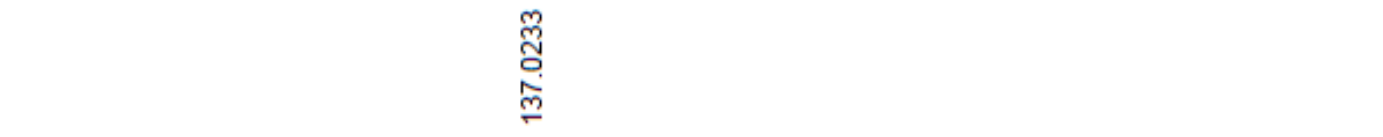 } \\
\hline 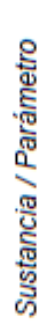 & 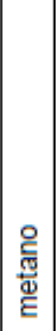 & 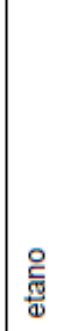 & \begin{tabular}{|l} 
읋 \\
음 \\
을
\end{tabular} & 윾 & 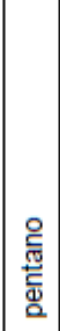 & 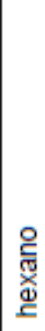 & 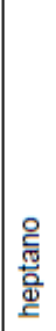 & $\begin{array}{l}\text { 음 } \\
\text { 용 }\end{array}$ & 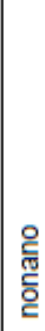 & $\begin{array}{l}\text { ్ㅠㅀ } \\
\text { dू }\end{array}$ & 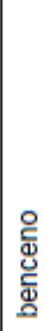 & 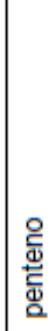 & 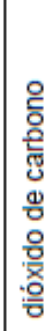 & 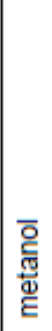 & $\begin{array}{l}\overline{\mathrm{D}} \\
\frac{\mathrm{F}}{\mathrm{s}}\end{array}$ & $\begin{array}{l}\overline{\mathrm{D}} \\
\frac{\mathrm{N}}{\mathrm{O}} \\
\text { 은 } \\
\stackrel{1}{1}\end{array}$ & 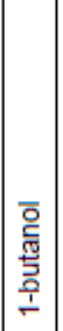 & 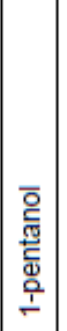 & 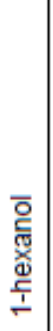 & 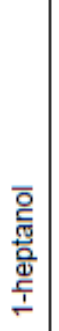 & 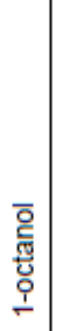 & 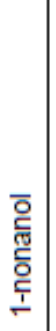 & 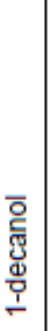 & 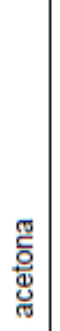 & 璺 & 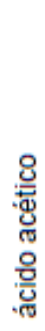 \\
\hline
\end{tabular}


Tabla 2: Valores de las constantes de la ecuación 13.

\begin{tabular}{|c|c|c|c|c|c|c|c|c|c|c|c|}
\hline \multicolumn{2}{|l|}{$\begin{array}{l}\text { Familia } \\
\text { química }\end{array}$} & \multicolumn{3}{|c|}{ n-alcanos } & \multicolumn{7}{|c|}{ n-alcoholes } \\
\hline \multirow{2}{*}{\multicolumn{2}{|c|}{ Parámetro }} & \multirow[b]{2}{*}{$e_{1}$} & \multirow[b]{2}{*}{$V_{1}$} & \multirow{2}{*}{$b_{2}$} & \multicolumn{3}{|c|}{$e_{1}$} & \multicolumn{3}{|c|}{$V_{1}$} & \multirow{2}{*}{$\begin{array}{c}b_{2} \\
\mathrm{M}_{\mathrm{w}} \geq 32\end{array}$} \\
\hline & & & & & $\begin{array}{l}M_{w} \leq 74 . \\
2\end{array}$ & $\begin{array}{l}74.2<\mathrm{M}_{\mathrm{w}} \leq 15 \\
8.3\end{array}$ & $\begin{array}{l}\mathrm{M}_{\mathrm{w}}>158 \\
.3\end{array}$ & $\begin{array}{l}M_{w} \leq 88 \\
2\end{array}$ & $\begin{array}{l}88.2<\mathrm{M}_{\mathrm{w}} \leq 14 \\
4.3\end{array}$ & $\begin{array}{l}M_{w}>144 . \\
3\end{array}$ & \\
\hline \multirow{2}{*}{$\begin{array}{l}\text { Constante } \\
s\end{array}$} & $\begin{array}{l}z \\
1 \\
\end{array}$ & 0.002 & -0.5987 & -0.289 & 0.0238 & -0.0034 & -0.0003 & $24 . \overline{9} 837$ & -5.184 & -1.7737 & -0.1103 \\
\hline & $\begin{array}{l}z \\
2 \\
\end{array}$ & $\begin{array}{c}- \\
0.378 \\
1\end{array}$ & $\begin{array}{c}- \\
346.978 \\
5\end{array}$ & $\begin{array}{c}- \\
100.804 \\
3 \\
\end{array}$ & $\begin{array}{c}- \\
1.1278\end{array}$ & 0.9245 & 0.0233 & $\begin{array}{c}574.557 \\
1\end{array}$ & -993.7328 & $\begin{array}{c}- \\
978.081 \\
8 \\
\end{array}$ & $\begin{array}{c}- \\
95.043 \\
7\end{array}$ \\
\hline
\end{tabular}

\section{EXTENSIÓN PARA MEZCLAS}

Se extiende el modelo a mezclas en especial en sistemas binarios, aplicando las siguientes reglas de mezclado: Regla de mezcla simple, regla de mezclado con un parámetro de interacción y regla de mezclado con dos parámetros de interacción.

Regla de Mezcla Simple: Regla de mezcla simple (Guo et al., 2001; Fan y Wang, 2006) son adaptadas usando los parámetros del modelo $P R \mu_{0}$, a partir de las ecuaciones 14 y 15 , así:

$$
\begin{aligned}
T_{m}^{\prime} & =\frac{R_{m}^{\prime} P}{\mu_{m}-b_{m}}-\frac{a_{m} \beta_{m}}{\mu_{m}^{2}+2 \mu_{m} b_{m}-b_{m}^{2}} \\
\theta_{m} & =\sum_{i=1}^{N C} x_{i} \theta_{i}
\end{aligned}
$$

Donde xi es la fracción molar y NC el número de sustancias.

Regla de mezclado con un parámetro de interacción: Se propone la siguiente regla de mezcla con un parámetro de interacción (Regla de mezcla 1):

$$
\begin{aligned}
& \varphi_{m}=\sum_{i=1}^{N C} \sum_{j=1}^{N C} x_{i} x_{j} \sqrt{\varphi_{i} \varphi_{j}}, \varphi=T_{d}, a, b, r_{c}, \alpha \\
& \beta_{m}=\sum_{i=1}^{N C} \sum_{j=1}^{N C} x_{i} x_{j} \sqrt{\beta_{i} \beta_{j}} \cdot\left(1-I_{i j}\right) \\
& \tau_{m}=\sum_{i=1}^{N C} x_{i} \tau_{i}, \tau=c_{0}, c, \mu_{c}
\end{aligned}
$$

En la ecuación 17, el parámetro de interacción $l_{i j}$ debe ser determinado a partir de condiciones experimentales. La regla de mezcla descrita anteriormente es una adaptación de la regla de mezclado propuesta por Khosharay et al. (2015).

Regla de mezclado con dos parámetros de interacción: Se propone la siguiente regla de mezcla con dos parámetros de interacción (Regla de mezcla 2):

$$
\begin{aligned}
& \delta_{m}=\sum_{i=1}^{N C} \sum_{j=1}^{N C} x_{i} x_{j}\left[\frac{\left(\delta_{i}\right)^{1 / 3}+\left(\delta_{j}\right)^{1 / 3}}{8}\right]^{3}, \delta=a, b, c_{0}, c, r_{c}, \beta \\
& T_{d m}=\sum_{i=1}^{N C} \sum_{j=1}^{N C} x_{i} x_{j}\left[\frac{\left(T_{d i}\right)^{1 / 3}+\left(T_{d j}\right)^{1 / 3}}{8}\right]^{3} \cdot\left(1-k_{i j}\right)
\end{aligned}
$$




$$
\alpha_{m}=\sum_{i=1}^{N C} \sum_{j=1}^{N C} x_{i} x_{j}\left[\frac{\left(\alpha_{i}\right)^{1 / 3}+\left(\alpha_{j}\right)^{1 / 3}}{8}\right]^{3} \cdot\left(1-W_{i j}\right)
$$

En las ecuaciones 20 y $21, k_{i j}$ y $W_{i j}$ son parámetros de interacción y se deben determinar a partir de datos experimentales. La regla de mezcla descrita anteriormente es una adaptación de la regla de mezclado propuesta por Khosharay et al. (2015) y el método de Teja y Rice (1981).

\section{RESULTADOS PARA SUSTANCIAS PURAS}

Se compara el modelo $P R \mu_{0}$ con diferentes modelos reportados en la literatura como lo son para fase líquida: Ltsou y Stiel (1973), Lewis y Squires (Mehrotra, 1991), Przezdziecki y Sridhar (Poling et al., 2001), Orrick y Erbar (Gardas y Coutinho, 2008); mientras que para la fase vapor las ecuaciones de: Chung et al. (1988), Lucas y Reichenberg (Satyro y Yarranton, 2010; Poling et al., 2001) y los modelos tipo ecuación de estado propuestos por Guo et al. (2001) y Fan y Wang (2006). Es importante aclarar que en el caso de alcanos y alcoholes, los parámetros $e_{1}, v_{1}, b_{2}$ se calculan mediante las expresiones generalizadas reportadas en la Tabla 2. Para el resto de las sustancias se utilizan los parámetros reportados en la Tabla 1. Las comparaciones se realizan a partir del cálculo de la desviación absoluta promedio (ver ecuación 12) y los resultados se muestran en la Tabla 3. La $T_{\min }$ y $T_{\text {máx }}$ corresponde al intervalo donde se encuentran los datos experimentales de viscosidad reportados y recomendados como aceptados por la DIPPR (Daubert, 1998).

En la Tabla 3 se infiere que para fase líquida el modelo de Lewis y Squires describe la viscosidad con una desviación promedio de $11.44 \%$ y para fase vapor el modelo de Lucas con $9.09 \%$, no obstante, el modelo $P R \mu_{0}$ desarrollado en esta investigación genera desviaciones del $6.35 \%$. Además, se muestra en esta Tabla que la correlación de Chung et al., presenta una desviación absoluta de $10.90 \%$ y de acuerdo con Poling et al. (2001) las desviaciones para este modelo se encuentran entre $0.2 \%$ y $12 \%$.

En este trabajo, para alcanos se obtienen desviaciones absolutas promedio con el modelo de Guo et al. (2001) de $44.13 \%$, estos resultados están conformes con lo reportado por Fan y Wang (2006), quienes indican que este modelo presenta desviaciones en el equilibrio líquido-vapor superiores al $32.4 \%$. Los modelos de Guo et al. (2001) y Fan y Wang (2006) son aplicables para la estimación de la viscosidad en sustancias no-polares, por tal motivo al extender estos modelos a sustancias polares producen desviaciones superiores al $133 \%$ para Guo et al. (2001) y 19\% para Fan y Wang (2006). En conclusión, no es adecuada la predicción de estos modelos en sustancias polares. Estos resultados están conformes a lo reportado por Bonyadi y Rostami (2017) y Khosharay et al. (2017).

Los perfiles de viscosidad en el equilibrio líquido-vapor se muestran en las Figura 1. Es importante aclarar que en la Figura 1 el punto donde las curvas de líquido y vapor saturado se encuentran corresponde a la viscosidad crítica (Poling et al., 2001). Además, se evalúa la predicción en condiciones de una sola fase y los resultados se muestran en la Figura 2. En las Figuras 1 y 2, el modelo $P R \mu_{0}$ desarrollado en esta investigación presenta una alta predicción de la viscosidad en el equilibrio líquido-vapor y en la región de una sola fase en un rango de presión entre 0.1 bar y 30 bar.
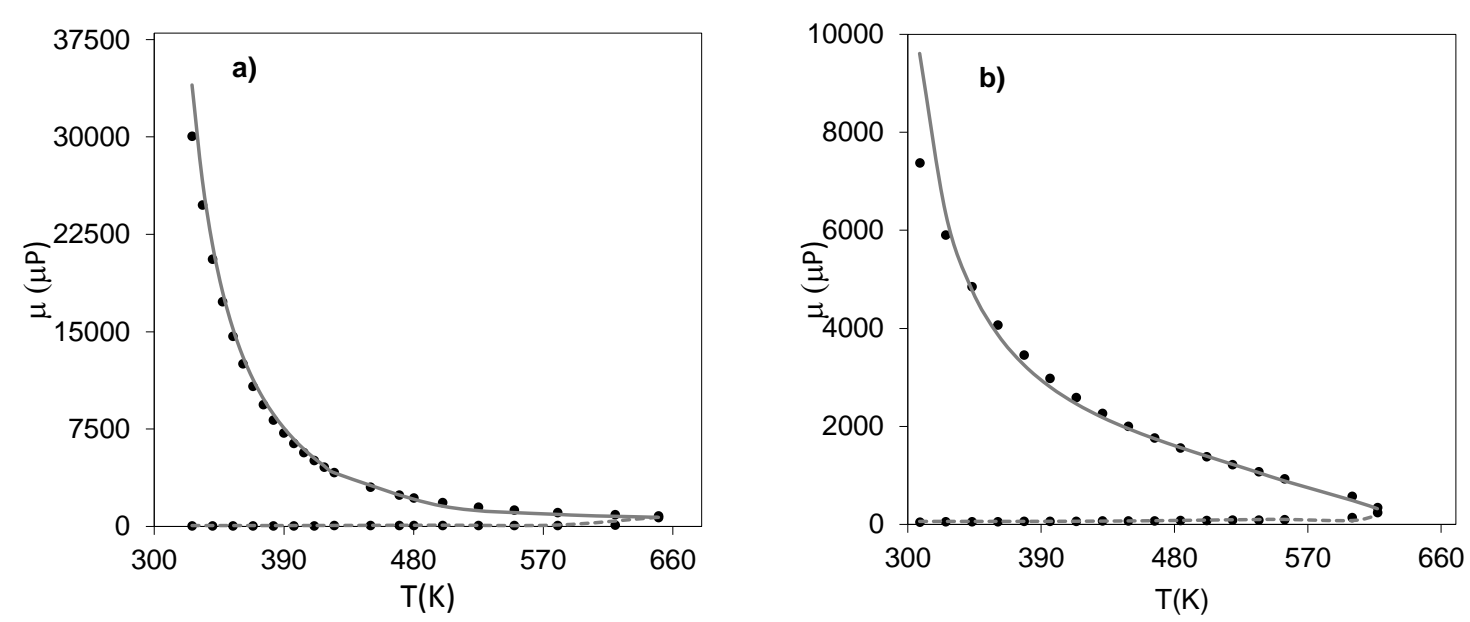

Fig. 1: Comparación de las viscosidades en el equilibrio líquido-vapor para el (a) 1-octanol y (b) n-decano, datos experimentales $(\bullet)$, calculado por el modelo $P R \mu \mu_{0}$ : líquido saturado (-), vapor saturado (- -). 

峞

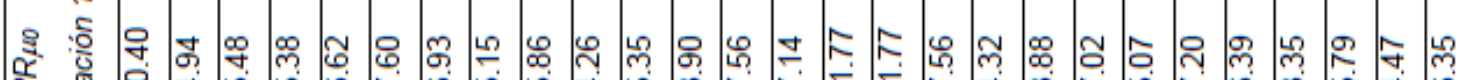

至

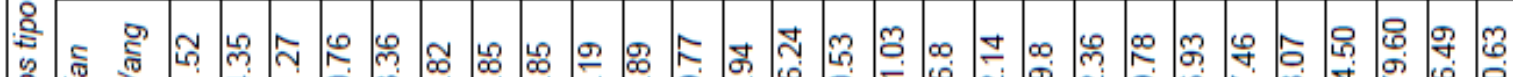

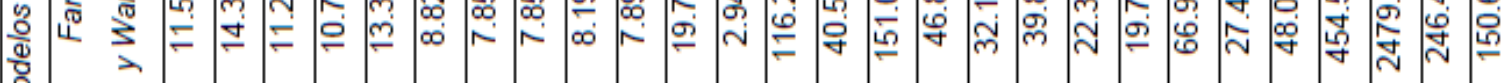

¿

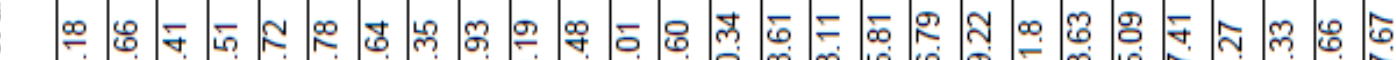

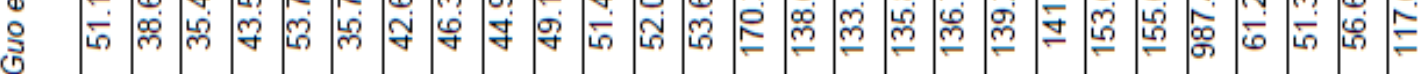

$\frac{\sqrt{3}}{3}$

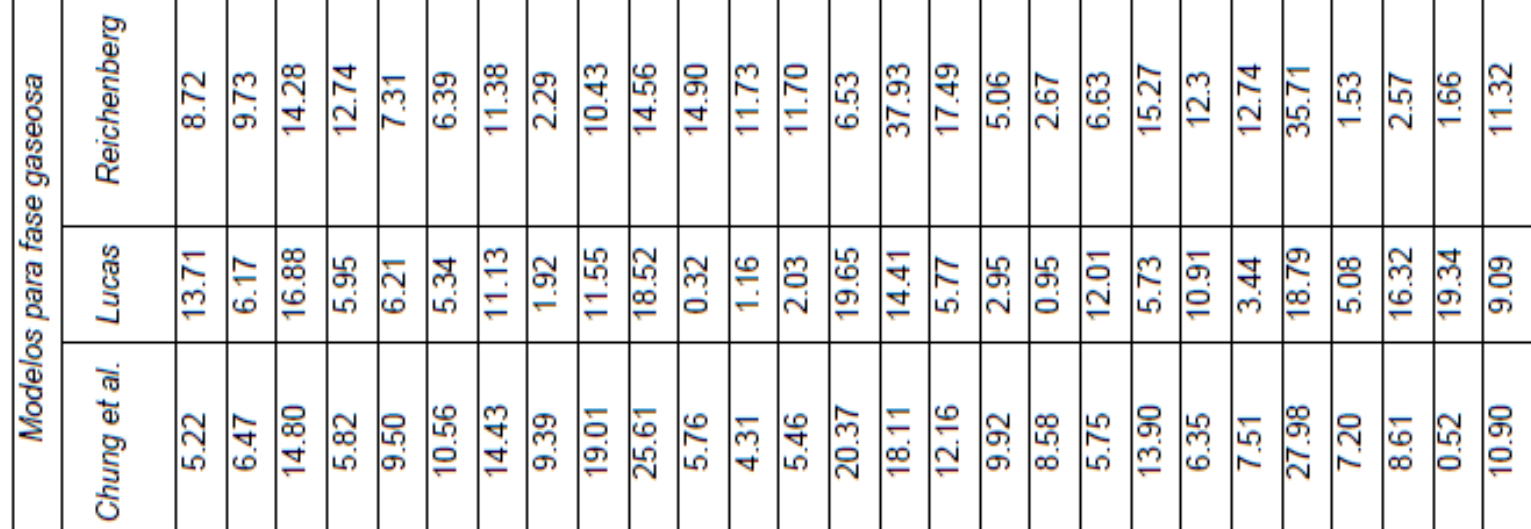

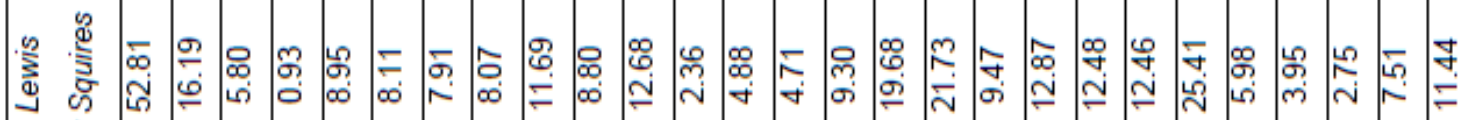

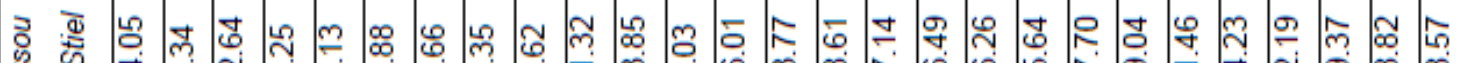
约 承

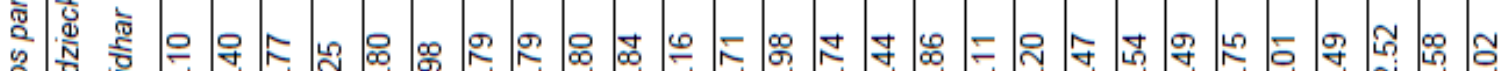
焉

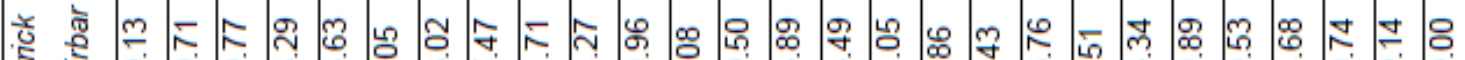

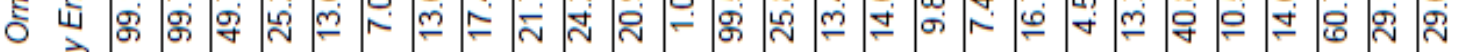

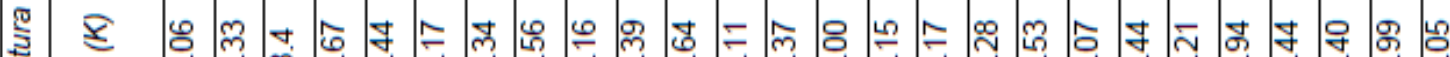

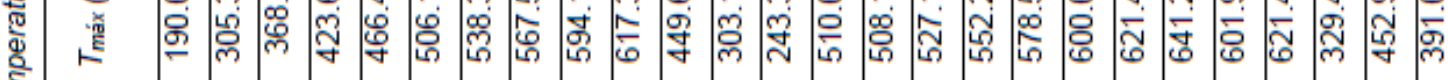
है

$\therefore$ 든 焉脿

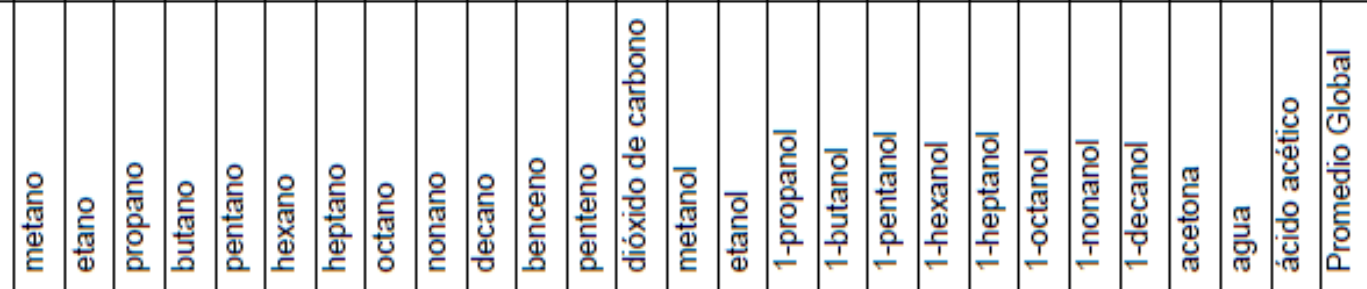



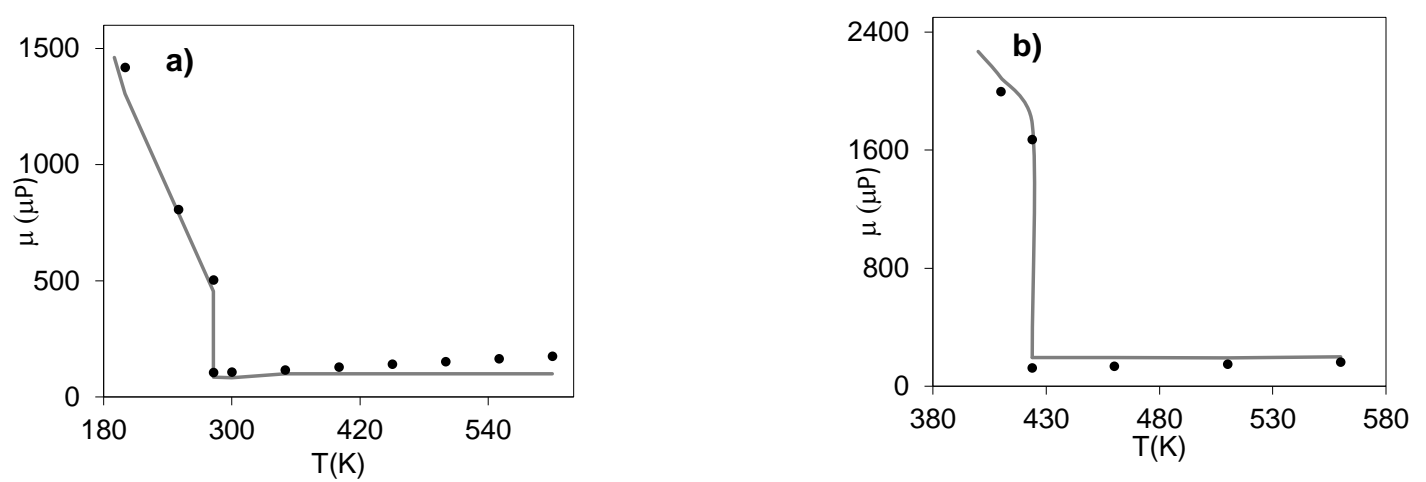

Fig. 2: Comparación de las viscosidades calculadas y experimentales del (a) etano y (b) etanol a 30 bar y 10 bar respectivamente, experimental $(\bullet), P R \mu_{0}(-)$.

En el caso del agua, la acetona y el ácido acético se obtienen desviaciones menores a 5.79\%. Por otro lado, sustancias como benceno, penteno y dióxido de carbono presentan desviaciones menores a $7.56 \%$. Estos resultados son similares a aquellos reportados en la literatura para diferentes tipos de correlaciones (Poling et al., 2001; Mehrotra, 1991).

Los resultados descritos en los párrafos anteriores incluyen solo las sustancias utilizadas en la determinación de parámetros del modelo (ver Tabla 1). Con el objetivo de mostrar la capacidad predictiva de las expresiones generalizadas de alcanos y alcoholes, se realizan cálculos predictivos entre undecano y eicosano, y entre 1undecanol y 1-eicosanol. En la Tabla 4 se muestra los resultados de las sustancias evaluadas. Para realizar la comparación, se seleccionan los datos recomendados y aceptados por la DIPPR (Daubert, 1998). Para cada sustancia se toman 30 datos experimentales que incluyen 15 datos de líquido saturado y 15 datos de vapor saturado en un intervalo de temperatura reducida entre 0.5 y 0.8 . En total 600 datos experimentales son utilizados en el proceso de predicción.

En la Tabla 4, las predicciones del modelo se pueden considerar adecuadas. La sustancia que menor desviación tiene es el heptadecano con 2.28\%, mientras que el 1-undecanol presenta desviaciones promedio de $35.09 \%$. Es importante aclarar, que para sustancias como 1-undecanol, 1-dodecanol, 1-hexadecanol y 1octadecanol el DIPPR reporta desviaciones superiores de 10\% aproximadamente para aquellos datos catalogados como recomendados (Daubert, 1998). Las predicciones realizadas con el modelo propuesto corresponden a una desviación promedio de $6.26 \%$ y $7.21 \%$ para alcanos y alcoholes respectivamente. En general la desviación absoluta promedio es de $8.88 \%$. Los valores máximos y la desviación relativa se encuentran dentro del error experimental. En la Figura 3 se muestra la calidad de la predicción de la viscosidad del $\mathrm{n}$-dodecano en el equilibrio líquido-vapor. De esta figura se concluye que el modelo $P R \mu_{0}$ realiza predicciones adecuadas en especial en temperaturas reducidas entre 0.50 y 0.80 .

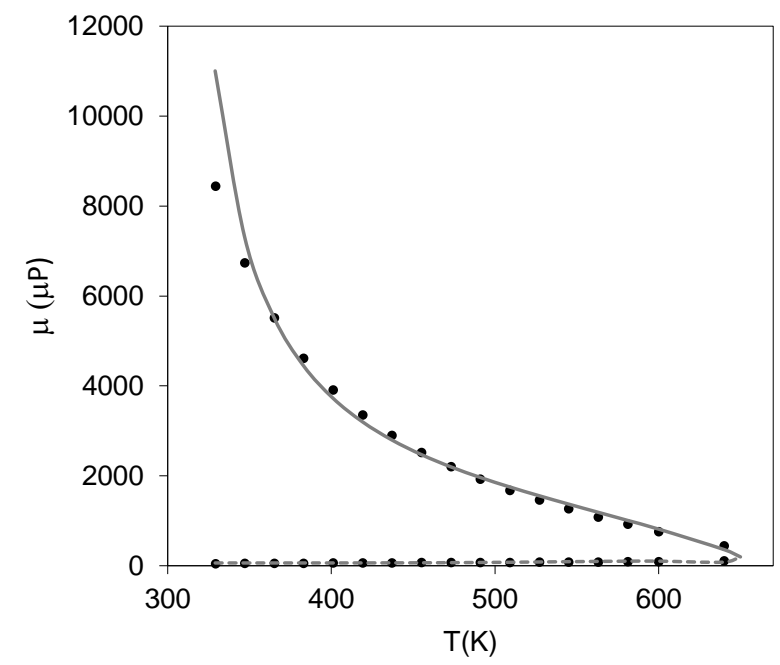

Fig. 3: Comparación de las viscosidades predichas en el equilibrio líquido-vapor para el n-dodecano, datos experimentales $(\bullet)$, calculado por el modelo $P R \mu$ : líquido saturado $(-)$, vapor saturado (- -). 
Tabla 4: Predicciones del modelo $P R \mu_{0}$ en el equilibrio líquido-vapor.

\begin{tabular}{|c|c|c|c|c|c|c|c|c|c|c|c|}
\hline \multicolumn{6}{|c|}{ Predicción n-alcanos } & \multicolumn{6}{|c|}{ Predicción n-alcoholes } \\
\hline \multirow{2}{*}{ Sustancias } & \multicolumn{2}{|c|}{$\begin{array}{l}\text { Intervalo de } \\
\text { Temperatura }\end{array}$} & \multirow{2}{*}{$\begin{array}{c}1 \% \Delta \\
\mu l\end{array}$} & \multirow{2}{*}{$\% \Delta \mu$} & \multirow{2}{*}{$\begin{array}{c}\text { máx } \\
1 \% \Delta \\
\mu l\end{array}$} & \multirow{2}{*}{ Sustancias } & \multicolumn{2}{|c|}{$\begin{array}{l}\text { Intervalo de } \\
\text { Temperatura }\end{array}$} & \multirow{2}{*}{$\begin{array}{l}\% \Delta \mu \\
I\end{array}$} & \multirow{2}{*}{$\% \Delta \mu$} & \multirow{2}{*}{$\begin{array}{l}\text { máx } \\
|\% \Delta \mu|\end{array}$} \\
\hline & $\begin{array}{l}T_{\min } \\
(K)\end{array}$ & $\underset{1}{T_{\text {máx }}(K}$ & & & & & $\begin{array}{l}T_{\min } \\
(K)\end{array}$ & $\underset{1}{T_{\text {máx }}(K}$ & & & \\
\hline undecano & 319.5 & 447.3 & 4.15 & -3.48 & 5.64 & 1-undecanol & 351.8 & 492.5 & $\begin{array}{c}35.0 \\
9\end{array}$ & $\begin{array}{c}- \\
18.47\end{array}$ & 63.89 \\
\hline dodecano & 329 & 460.6 & 5.82 & 5.14 & 9.32 & 1-dodecanol & 359.7 & 503.6 & 21.3 & -9.76 & 41.68 \\
\hline tridecano & 337.5 & 472.5 & 4.2 & -0.09 & 6.82 & 1-tridecanol & 367 & 513.8 & 3.44 & 3.81 & 8.93 \\
\hline tetradecano & 346.5 & 485.1 & 4.66 & 3.15 & 4.99 & 1-tetradecanol & 373.5 & 522.9 & 3.46 & 3.4 & 9.37 \\
\hline pentadecano & 354 & 495.6 & 3.13 & 1.96 & 5.08 & 1-pentadecanol & 379.5 & 531.3 & 6.2 & 6.55 & 17.5 \\
\hline hexadecano & 361.5 & 506.1 & 3.3 & 2.91 & 5.24 & 1-hexadecanol & 385 & 539 & 24.5 & -9.46 & 48.1 \\
\hline heptadecano & 368 & 515.2 & 2.28 & 2.02 & 4.17 & 1-heptadecanol & 390 & 546 & 6.48 & 8.55 & 17.96 \\
\hline octadecano & 373.5 & 522.9 & 3.65 & 2.04 & 6.63 & 1-octadecanol & 395 & 553 & $\begin{array}{c}27.0 \\
2\end{array}$ & $10 \overline{-}$ & 55.38 \\
\hline nonadecano & 379 & 530.6 & 3.73 & 0.33 & 5.12 & 1-nonadecanol & 399.5 & 559.3 & 5.13 & -0.02 & 11.17 \\
\hline eicosano & 384 & 537.6 & 5.02 & 0.17 & 5.97 & 1-eicosanol & 404.5 & 566.3 & 5.02 & -0.28 & 15.28 \\
\hline Promedio & & & 3.99 & 1.42 & & Promedio & & & $\begin{array}{c}13.7 \\
6\end{array}$ & -2.60 & \\
\hline
\end{tabular}

\section{RESULTADOS PARA MEZCLAS}

Se evaluaron tres reglas de mezcla para 15 sistemas binarios que incluyen mezclas alcano+alcano, alcohol+alcohol, alcohol+alcano, benceno+octano, pentanol+benceno y acetona+etanol y los resultados se muestran en la Tabla 5 y Figura 4.

En la Tabla 5, la regla de mezcla simple para sistemas alcano+alcano produce desviaciones promedio de $20.21 \%$ y se verifica en la Figura 4. Fan y Wang (2006) reporta desviaciones absolutas para este tipo de sistemas de $11.84 \%$. Para este mismo sistema, la regla de mezcla con un parámetro de interacción presenta desviaciones de $9.44 \%$ y de acuerdo con lo reportado por Khosharay et al. (2015) las desviaciones promedio absolutas son de $14.74 \%$.
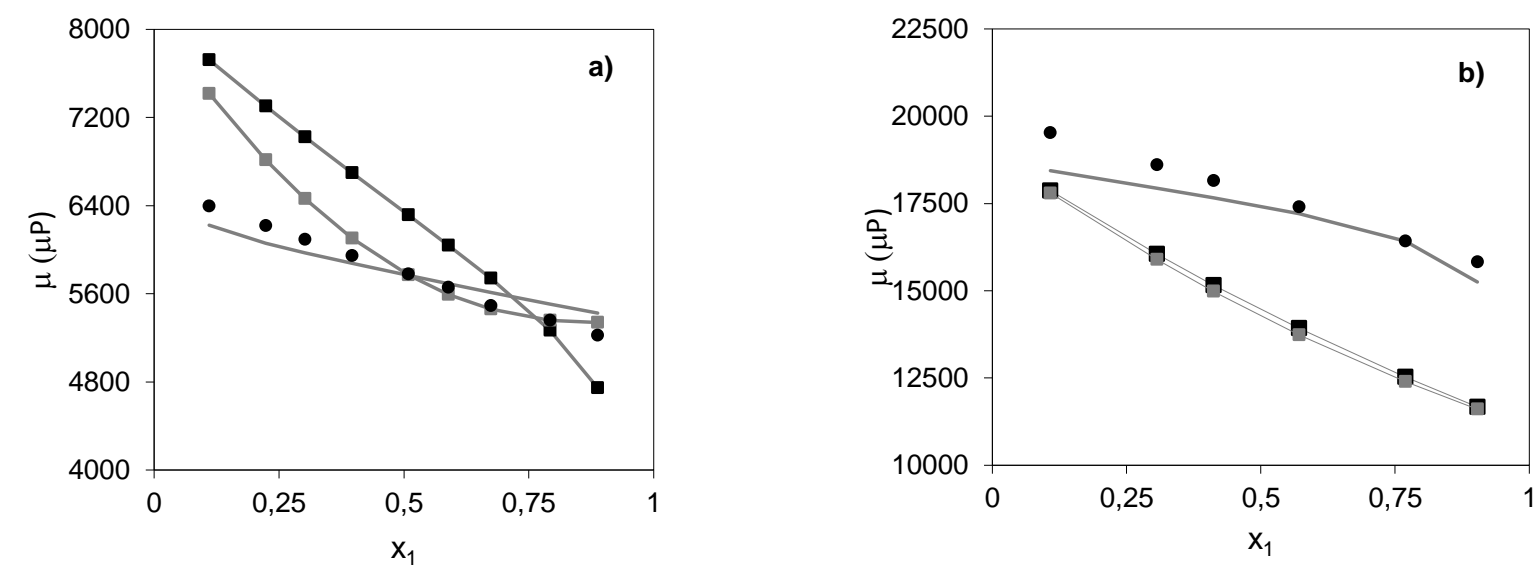

Fig. 4: Comparación de las viscosidades de mezcla calculadas y experimentales del sistema (a) octano+nonano y (b) 1propanol+1-butanol: (•) experimental; (-m-) Regla de mezcla simple;(-m-) Regla de mezcla 1; (-) Regla de mezcla 2 a $\mathrm{T}=298.15 \mathrm{~K}$ y $\mathrm{P}=1$ bar.

Para sistemas del tipo alcohol+alcohol la regla de mezcla simple produce desviaciones menores a $21.52 \%$, por tal motivo no es recomendable el uso de esta regla de mezclado para estos sistemas. Las reglas de mezcla con uno y dos parámetros de interacción producen desviaciones menores a $13.79 \%$ y $6.14 \%$, respectivamente y su comportamiento se muestra en la Figura 4. Por otro lado, para sistemas alcohol+alcano, la regla de mezcla simple presenta desviaciones de $15.50 \%$, mientras que las reglas de uno y dos parámetros son de $5.69 \%$ y $3.14 \%$ respectivamente. En general, la regla de mezcla con dos parámetros de interacción produce resultados satisfactorios con desviaciones promedio de $3.85 \%$ y su comportamiento es verificado en la Figura 4. 
Tabla 5: Comparación del porcentaje de desviación absoluta en el cálculo de viscosidades de mezclas en sistemas binarios a una presión de 1 bar y temperaturas entre $298.15 \mathrm{~K}$ y $313.15 \mathrm{~K}$.

\begin{tabular}{|c|c|c|c|c|c|c|c|c|}
\hline \multirow[b]{2}{*}{ Mezcla } & \multirow[b]{2}{*}{$x_{1}$} & \multirow{2}{*}{$\begin{array}{c}\begin{array}{c}\text { Regla } \\
\text { de mezcla } 1\end{array} \\
l_{i j}\end{array}$} & \multicolumn{2}{|c|}{$\begin{array}{c}\text { Regla } \\
\text { de mezcla } 2\end{array}$} & \multicolumn{3}{|c|}{$|\% \Delta \mu|$} & \multirow[b]{2}{*}{ Ref. } \\
\hline & & & $k_{i j}$ & $W_{i j}$ & $\begin{array}{c}\text { Regla } \\
\text { de } \\
\text { Mezcla } \\
\text { simple }\end{array}$ & $\begin{array}{c}\text { Regla } \\
\text { de } \\
\text { mezcla } \\
1 \\
\end{array}$ & $\begin{array}{c}\text { Regla } \\
\text { de } \\
\text { mezcla } \\
2 \\
\end{array}$ & \\
\hline hexano(1)+dodecano(2) & $\begin{array}{l}0.0000- \\
1.0000 \\
\end{array}$ & 0.7413 & 0.07752 & -0.9823 & 12.79 & 4.81 & 1.08 & Chevalier et al. (1990) \\
\hline octano (1)+nonano (2) & $\begin{array}{l}0.1101- \\
0.8879\end{array}$ & 0.9737 & 0.1542 & -0.9929 & 17.84 & 4.57 & 2.58 & Chevalier et al. (1990) \\
\hline heptano(1)+dodecano(2) & $\begin{array}{l}0.2001- \\
0.7999\end{array}$ & 0.9770 & -0.0316 & 0.6671 & 12.93 & 7.15 & 1.90 & Chevalier et al. (1990) \\
\hline dodecano(1)+hexadecano(2) & $\begin{array}{l}0.2028- \\
0.7998\end{array}$ & -0.7080 & 0.3345 & 0.9198 & 37.28 & 21.25 & 4.83 & Chevalier et al. (1990) \\
\hline 1-heptanol(1)+hexano(2) & $\begin{array}{l}0.0308- \\
0.9642\end{array}$ & 0.9316 & 0.0549 & -0.7764 & 6.97 & 5.02 & 3.74 & Sastry y Raj (1996) \\
\hline 1-heptanol(1)+heptano(2) & $\begin{array}{l}0.0373- \\
0.9699\end{array}$ & 0.9981 & 0.0690 & -0.6461 & 9.50 & 5.67 & 4.84 & Sastry y Raj (1996) \\
\hline 1-butanol(1)+hexano(2) & $\begin{array}{l}0.0733- \\
0.9029 \\
\end{array}$ & -0.8230 & 0.0638 & 0.2576 & 37.28 & 9.29 & 1.45 & Franjo et al. (1995) \\
\hline 1-hexanol(1)+hexano(2) & $\begin{array}{l}0.0507- \\
0.8673 \\
\end{array}$ & 0.6440 & -0.0054 & -0.0065 & 8.28 & 2.79 & 2.55 & Franjo et al. (1995) \\
\hline 1-propanol(1)+1-butanol(2) & $\begin{array}{c}0.1080- \\
0.892 \\
\end{array}$ & 0.0008 & 0.0012 & -0.4124 & 18.07 & 6.08 & 4.26 & Shan y Asfour (1998) \\
\hline 1-propanol(1)+1-pentanol(2) & $\begin{array}{l}0.0000- \\
1.0000 \\
\end{array}$ & 0.9898 & -0.0015 & -0.5704 & 23.08 & 22.80 & 7.03 & Shan y Asfour (1998) \\
\hline 1-pentanol(1)+1-octanol(2) & $\begin{array}{l}0.0000- \\
1.0000 \\
\end{array}$ & 0.9859 & 0.1030 & -0.9983 & 16.01 & 10.52 & 6.64 & Shan y Asfour (1998) \\
\hline 1-heptanol(1)+1-octanol(2) & $\begin{array}{l}0.0000- \\
1.0000\end{array}$ & 0.9745 & -0.0007 & 0.3113 & 28.91 & 15.76 & 6.62 & Shan y Asfour (1998) \\
\hline benceno (1)+octano (2) & $\begin{array}{l}0.2539- \\
0.6694 \\
\end{array}$ & 0.9611 & 0.2475 & -0.80226 & 18.05 & 17.07 & 2.65 & $\begin{array}{c}\text { Dymon y Young } \\
(1981)\end{array}$ \\
\hline pentanol $(1)+$ benceno $(2)$ & $\begin{array}{l}0.0000- \\
1.0000 \\
\end{array}$ & -0.9989 & -0.0173 & 0.4844 & 16.12 & 6.50 & 2.06 & $\begin{array}{c}\text { Tsierkezos et al. } \\
(2000)\end{array}$ \\
\hline acetona (1) + etanol (2) & $\begin{array}{l}0.0000- \\
1.0000\end{array}$ & 0.0213 & -0.0190 & 0.7865 & 16.85 & 15.41 & 5.56 & Chen y Tu (2005) \\
\hline Promedio & & & & & 18.66 & 10.31 & 3.85 & \\
\hline
\end{tabular}

Los coeficientes de interacción reportados en la Tabla 5 son obtenidos utilizando la función objetivo dado por la Ecuación 13. Para la regla de mezcla 1 , los valores estimados de $l_{i j}$ son altos porque la regla de mezclado utiliza únicamente este coeficiente de interacción para el parámetro $\beta$, los otros dos parámetros no tienen coeficiente de interacción por lo tanto $l_{i j}$ debe compensar todos los errores de mezclado. Por otro lado, la regla de mezcla 2 los valores de los coeficientes de interacción son similares aquellos reportados en la literatura (Khosharay et al., 2015; Khosharay et al., 2017). Además, es posible calcular combinaciones de $\mathrm{k}_{\mathrm{ij}}$ y $\mathrm{W}_{\mathrm{ij}}$ con valores diferentes pero que pueden llevar a desviaciones similares. Por ejemplo, para benceno+octano los valores de la Tabla 5 son $\mathrm{k}_{\mathrm{ij}}=0.2475$ y $\mathrm{W}_{\mathrm{ij}=}=-0.80226$ y la desviación de $2.65 \%$, mientras que otros valores estimados son: $\mathrm{k}_{\mathrm{ij}}=0.1165$ y W $\mathrm{Wij}=0.02012$ con desviación de $5.56 \%$. En ambos casos, corresponden a una buena representación de la mezcla.

\section{CONCLUSIONES}

A partir de los resultados obtenidos, se pueden extraer las siguientes cuatro conclusiones: 1) El modelo de viscosidad del tipo ecuación cúbica de estado de Peng-Robinson en el intervalo de trabajo de cada sustancia presenta la menor desviación absoluta en comparación con los otros modelos evaluados en esta investigación, tanto para sustancias polares y no polares, describiendo en un solo modelo la fase líquida y vapor; 2) El modelo realiza predicciones satisfactorias en $n$-alcanos entre undecano y eicosano y $n$-alcoholes entre 1-undecanol y 1-eicosanol; 3) Se confrontó los resultados obtenidos con diferentes ecuaciones propuestas en la literatura siendo el modelo de viscosidad para fase vapor de Lucas y Lewis y Squires para fase líquida quienes producen la menor desviación, sin embargo, el modelo tipo ecuación cúbica de estado de Peng-Robinson genera bajas desviaciones en comparación con estos modelos; 4) La viscosidad en mezclas se evaluó utilizando tres enfoques: regla de mezcla simple, regla de mezcla con uno y dos parámetros de interacción donde la regla de mezcla de dos parámetros es quien mejor describe la viscosidad con desviaciones absolutas promedio de $3.85 \%$. 


\section{AGRADECIMIENTOS}

Los autores agradecen a la Universidad Pontificia Bolivariana y al programa de COLCIENCIAS "Doctorados Nacionales-convocatoria 727 del 2015", por su financiación y apoyo en la realización de la presente investigación.

\section{NOTACIÓN}

\section{Símbolos}

$a_{c}, b_{c}, r_{c}, \mathrm{R}^{\prime}=$ parámetros de la ecuación de estado de Peng-Robinson (ver ecuaciones $2-5$ )

$c=$ parámetros de corrección de la viscosidad (ver ecuaciones $9-11$ )

EoS $=$ ecuación de estado

$e, a, b, v=$ constantes del modelo $P R \mu_{0}$ (ver ecuaciones $6-11$ )

$k, I, W=$ coeficientes de interacción (ver ecuaciones 17, 20 y 21)

$M_{w}=$ peso molecular en $\mathrm{g} / \mathrm{mol}$

$\mathrm{N}=$ número total de datos experimentales

$N C=$ número de sustancias en una mezcla

$P=$ presión (bar)

$\mathrm{PR}$ = ecuación de estado de Peng-Robinson

PT = ecuación de estado de Patel-Teja

$P R \mu_{0}=$ modelo de viscosidad desarrollado a partir de la ecuación de estado de Peng-Robinson

$P V T=$ diagrama termodinámico de volumen molar

$\mathrm{R}=$ constante universal de los gases ideales

$T=$ temperatura $(\mathrm{K})$

$T \mu P=$ diagrama termodinámico de viscosidad

$T_{d}=$ temperatura especifica debida a la corrección de viscosidad definida como $T_{d}=0.45 \cdot T_{c}$

$T^{\prime}=$ temperatura supuesta definida como $T^{\prime}=\left|T-T_{d}\right|$

$V=$ volumen molar

$x=$ fracción molar

$Z=$ factor de compresibilidad

$\mathrm{Z}=$ constante de la ecuación 13

\section{Letras Griegas}

$\alpha=$ parámetro similar a las funciones alfa de las ecuaciones $P V T$ (ver ecuación 6)

$\beta=$ parámetro del modelo $P R \mu_{0}$ (ver ecuación 7)

$\% \Delta=$ porcentaje de desviación

$\theta, \tau, \varphi, \delta=$ parámetros de mezcla (ver ecuaciones $15,16,18,19$ y 21 )

$\mu=$ viscosidad en $\mu \mathrm{P}$ (micropoise)

\section{Abreviaciones}

DIPPR = Instituto de Diseño para la Investigación de Propiedades Físicas

F.O. = función objetivo

MS Excel $=$ Microsoft Excel

NIST = Instituto Nacional de Estándares y Tecnología

Súper/sub índices

$\mathrm{cal}=$ calculado

$c=$ condiciones críticas

$\exp =$ experimental

$\mathrm{m}=$ mezcla

$M O D E L O=$ viscosidad obtenida luego de resolver la ecuación 1

máx = máximo

mín =mínimo

$r=$ propiedad reducida

$0=$ parámetro del modelo $P R \mu_{0}($ ver ecuación 9$)$

1 - 3 = constantes del modelo $P R \mu_{0}($ ver Tabla 1$)$ 


\section{REFERENCIAS}

Abudour, A.M., S.A. Mohammad, R.L. Robinson y K.A.M. Gasem, Volume-translated Peng-Robinson equation of state for saturated and single-phase liquid densities, Fluid Phase Equilibria, 335, 74-87 (2012)

Bonyadi, M. y M. Rostami, A new viscosity model based on Soave-Redlich-Kwong equation of state, Fluid Phase Equilibria, 451, $40-47$ (2017)

Cardona-Palacio, L.F., Cálculo de la viscosidad de hidrocarburos a partir de la ecuación cúbica de estado de JarrahianHeidaryan, Revista Mexicana de Ingeniería Química, 15(3), 1019-1025 (2016)

Cardona-Palacio, L.F., Nueva correlación generalizada para estimar la presión de vapor, Revista científica, 2(25), 280289 (2016)

Chapra, S.C. y R.P. Canale, Métodos numéricos para ingenieros, 5ª Ed., McGraw-Hill, México (2007)

Chen, H.W. y C.H. Tu, Densities, viscosities, and refractive indices for binary and ternary mixtures of acetone, ethanol, and 2, 2, 4-trimethylpentane, Journal of Chemical \& Engineering Data, 50(4), 1262-1269 (1981)

Chevalier, J.L.E., P.J. Petrino e Y.H. Gaston-Bonhomme, Viscosity and density of some aliphatic, cyclic, and aromatic hydrocarbons binary liquid mixtures, Journal of Chemical and Engineering Data, 35(2), 206-212 (1990)

Chung, T.H., M. Ajlan, L.L. Lee y K.E. Starling, Generalized multiparameter correlation for nonpolar and polar fluid transport properties, Industrial \& Engineering Chemistry Research, 27(4), 671-679 (1988)

Daubert, T.E., Evaluated equation forms for correlating thermodynamic and transport properties with temperature, Industrial \& Engineering Chemistry Research, 37(8), 3260-3267 (1998)

Dymond, J.H. y K.J. Young, Transport properties of nonelectrolyte liquid mixtures-V. Viscosity coefficients for binary mixtures of benzene plus alkanes at saturation pressure from 283 to $393 \mathrm{~K}$, International Journal of Thermophysics, 2(3), 237-247 (1981)

Fan, T.B. y L.S. Wang, A viscosity model based on Peng-Robinson equation of state for light hydrocarbon liquids and gases, Fluid Phase Equilibria, 247(1-2), 59-69 (2006)

Fernández, G., J. Vrabec y H. Hasse, A molecular simulation study of shear and bulk viscosity and thermal conductivity of simple real fluids, Fluid Phase Equilibria, 221(1-2), 157-163 (2004)

Franjo, C., C.P. Menaut, E. Jiménez, J.L. Legido y M.I. Paz Andrade, Viscosities and densities of octane+butan-1-ol, hexan-1-ol, and octan-1-ol at 298.15 K, Journal of Chemical and Engineering Data, 40(4), $992-994$ (1995)

García, S.B., M.R. Estrada y G.I. Silva, Correlación para el Cálculo de Viscosidades de 1-Alcoholes, Conciencia Tecnológica, (34), 66-67 (2007)

Gardas, R.L. y J.A.P. Coutinho, A group contribution method for viscosity estimation of ionic liquids, Fluid Phase Equilibria, 266(1-2), 195-201 (2008)

Guo, X.Q., C.Y. Sun, S.X. Rong, G.J. Chen y T.M. Guo, Equation of state analog correlations for the viscosity and thermal conductivity of hydrocarbons and reservoir fluids, Journal of Petroleum Science and Engineering, 6 (1), $251-27$ (2001)

Guo, X.Q., L.S. Wang, S.X. Rong y T.M. Guo, Viscosity model based on equations of state for hydrocarbon liquids and gases, Fluid Phase Equilibria, 139, 405-421 (1997)

Jarrahian, A. y E. Heidaryan, A new cubic equation of state for sweet and sour natural gases even when composition is unknown, Fuel, 134, 333-342 (2014)

Khosharay, S., M. Pierantozzi y G. Di Nicola, Modeling investigation on the viscosity of pure refrigerants and their liquid mixtures by using the Patel-Teja viscosity equation of state, International Journal of Refrigeration, 85, 255-267 (2017)

Khosharay, S., R. Karimi y K. Khosharay, Modeling the viscosity for ( $\mathrm{nC5}+\mathrm{nC} 8)$, (nC5+nC10), (nC8+nC10) and $(\mathrm{nC5}+\mathrm{nC} 8+\mathrm{nC} 10)$ systems with Peng-Robinson viscosity equation of state, Periodica Polytechnica Chemical Engineering, 60(4), 259-265 (2015)

Lasdon, L.S., A.D. Waren, A. Jain y M. Ratner, Design and testing of a generalized reduced gradient code for nonlinear programming, ACM Transactions on Mathematical Software, 4(1) 34-50 (1978)

Lawal, A.S., Prediction of vapor and liquid viscosities from the Lawal-Lake-Silberberg equation of state, 5th Symposium on Enhanced Oil Recovery, Tulsa, EUA, Enero 20-23 Abril (1986)

Letsou, A. y L. Stiel, Viscosity of Saturated Nonpolar Liquids at Elevated Pressures, AIChE Journal, 409-411 (1973)

Linstrom, P.J. y W.G. Mallard, Eds., NIST Chemistry WebBook, NIST Standard Reference Database Number 69, National Institute of Standards and Technology, Gaithersburg, EUA (2018)

Little, J.E. y H.T. Kennedy, A correlation of the viscosity of hydrocarbon systems with pressure, temperature and composition, Society of Petroleum Engineers Journal, 8(02), 157-162 (1968)

Llovell, F., R.M. Marcos y L.F. Vega, Free-Volume Theory Coupled with Soft-SAFT for Viscosity Calculations: Comparison with Molecular Simulation and Experimental Data, The Journal of Physical Chemistry, B 117(27), 8159-8171 (2013)

Márquez-Baños, E.V., J.J. Valencia-López, O. García-Aranda y C. Heard, Determinación Computacional del Coeficiente 
de Transferencia de Calor en Calentadores Eléctricos de Flujo Continuo, mediante Dinámica de Fluidos Computacional, Información Tecnológica, 27(5), 151-162 (2016)

Mehrotra, A.K., Generalized one-parameter viscosity equation for light and medium liquid hydrocarbons, Industrial \& Engineering Chemistry Research, 30(6), 1367-1372 (1991)

Nazarzadeh, M. y M. Moshfeghian, New volume translated PR equation of state for pure compounds and gas condensate systems, Fluid Phase Equilibria, 337, 214-223 (2013)

NIST Chemistry WebBook. Propiedades termofísicas de sistemas fluidos, EUA (2017)

Novak, L.T., Predicting natural gas viscosity with a mixture viscosity model for the entire fluid region, Industrial \& Engineering Chemistry Research, 52(45), 16014-16018 (2013)

Ortega, A.F., A.O. Pérez y A.E. López, Modelo Semifísico de Base Fenomenológica del Proceso Continuo de Fermentación Alcohólica, Información Tecnológica, 27(1), 21-32 (2016)

Peng, D.Y. y D.B. Robinson, A new two-constant equation of state, Industrial \& Engineering Chemistry Fundamentals, 15(1), 59-64 (1976)

Poling, B.E., J.M. Prausnitz y J.P. O'Connell, The properties of gases and liquids, Vol. 5, New York, Mcgraw-Hill (2001)

Quiñones-Cisneros, S.E., C.K. Zéberg-Mikkelsen y E.H. Stenby, Friction theory prediction of crude oil viscosity at reservoir conditions based on dead oil properties, Fluid Phase Equilibria, 212(1), 233-243 (2003)

Qunfang, L. y H. Yu-Chun, Correlation of viscosity of binary liquid mixtures, Fluid Phase Equilibria, 154(1), 153-163 (1999)

Sastry, N. V. y M.M. Raj, Densities, speeds of sound, viscosities, dielectric constants, and refractive indices for 1-heptanol+ hexane and+ heptane at 303.15 and $313.15 \mathrm{~K}$, Journal of Chemical \& Engineering Data, 41 (3), $612-618$ (1996)

Satyro, M.A. y H.W. Yarranton, Expanded fluid-based viscosity correlation for hydrocarbons using an equation of state, Fluid Phase Equilibria, 298(1), 1-11 (2010)

Shan, Z. y A.F.A. Asfour, Viscosities and densities of eight binary 1-alkanol systems at 308.15 and $313.15 \mathrm{~K}$, Fluid Phase Equilibria, 143(1-2), 253-262 (1998)

Shukla, D., S. Singh, S. Parveen, M. Gupta y J.P. Shukla, A corresponding state model for the prediction of the viscosity of pure liquids and liquid mixtures, Journal of Molecular Liquids, 136(1), 111-116 (2007)

Teja, A.S. y P. Rice, Generalized corresponding states method for the viscosities of liquid mixtures, Industrial \& Engineering Chemistry Fundamentals, 20(1), 77-81 (1981)

Tsierkezos, N.G., M.M. Palaiologou e I.E. Molinou, Densities and viscosities of 1-pentanol binary mixtures at $293.15 \mathrm{~K}$, Journal of Chemical \& Engineering Data, 45(2), 272-275 (2000)

Wu, X., C. Li y W. Jia, An improved viscosity model based on Peng-Robinson equation of state for light hydrocarbon liquids and gases, Fluid Phase Equilibria, 380, 147-151 (2014)

Xuan, A.G., Y.X. Wu y otros cuatro autores, Correlation of viscosities for alkane, aromatic and alcohol family at high pressure by modified Tait equation, Chinese Journal of Chemical Engineering, 14(3), 364-370 (2006) 